\title{
System Development of an Experimental Rocket and an Autonomous Free Falling Mobile Robot
}

\author{
Yuito Mori ${ }^{a}$, Naoki Ogushi ${ }^{b}$, Tokunari Nishimura ${ }^{c}$ and Minoru Sasaki ${ }^{d}$ \\ Department of Human and Information Systems Engineering, faculty of Engineering, Gifu University, \\ Gifu, Japan 1-1 Yanagido, Gifu, 501-1193 \\ ap3128031@edu.gifu-u.ac.jp, bo312006@edu.gifu-u.ac.jp, ctokunari@ballade.plala.or.jp, \\ dsasaki@gifu-u.ac.jp
} Keywords: experimental rocket, system development, free falling mobile robot, autonomous
navigation, GPS

\begin{abstract}
This paper presents the development of a small experimental rocket for the National Launching Campaign held by the DGAEM in France since 2009. The campaign organized by CNES was conducted by the French Association of Planete Sciences. The rocket design was developed by Space Club Gifu and Sasaki Lab at Gifu University. The main mission is the autonomous navigation of a quasi-satellite maneuvering with a parachute and two propellers to a target point using GPS data. The rocket is $2 \mathrm{~m}$ long, $150 \mathrm{~mm}$ in diameter, weight $11.4 \mathrm{~kg}$ and made entirely of CFRP. With a solid rocket motor provided by CNES, the rocket can reach an altitude of $840 \mathrm{~m}$. Onboard are a pressure sensor, accelerometer, GPS and two video cameras. The video cameras begin monitoring at launch. The acceleration, velocity and position were recorded for later comparison to simulation data. While the rocket launch and quasi-satellite deployment were successful, strong winds prevented the latter from maneuvering to the target point. All components were recovered intact and all recorded data were available for analysis.
\end{abstract}

\section{Introduction}

The French Association of Planete Sciences, under the auspices of CNES (Centre National D'Etudes Spatiales), has been organizing launching campaign for university students and young engineers since 1962 [1]. Every year, they gather for an experimental launching campaign call "La Campagne National de Lancement". The launching campaign brings the clubs' efforts to fruition, but perhaps more importantly, provides a framework to learn and experience how a methodical experimental approach combined with rigorous project management works to attain a final goal. CNES and the Association of Planete Sciences provided comprehensive documentation on safety regulations [2], specifications of the provided solid rocket motor, the telemetry system [3], and requirements the rocket must meet. Our group, consisting of members from Space Club Gifu and the Sasaki Lab, has been participating in this launching campaign since $2005[4,5,6]$.

Our newest rocket, named UCG-10. UCG are Space Club Gifu's initials in Japanese. The UCG-10 saw its first launch in 2010. It is a single stage rocket made entirely of CFRP (Carbon Fiber Reinforced Plastic) and has several functions and mechanisms:

- It carries a quasi-satellite equipped with GPS, a parachute, a microcomputer and two propellers.

- It is controlled by five microcomputers mounted on several circuit boards.

- It has two onboard cameras to monitor rocket motor operation and quasi-satellite deployment.

- It features a new ejection system for the quasi-satellite using two side doors.

Our club is a regular participant in the launching campaign. We strive to improve our rocket design, and increase the variety of our missions each year. This year mission objectives are:

(1) The recording of pressure, acceleration and GPS data for later comparison with simulation results.

(2) Using two onboard cameras to monitor the deployment of the quasi-satellite and its parachute.

(3) The transmission and reception of telemetry from the rocket in flight.

(4) The autonomous navigation of the quasi-satellite to a target destination using onboard GPS. 


\section{The UCG-10 Rocket}

Design of the UCG-10 rocket. The UCG-10 rocket stands $2 \mathrm{~m}$ tall, is $150 \mathrm{~mm}$ in diameter and weighs $11.4 \mathrm{~kg}$. Table. 1 summarizes these measurements. The rocket can reach an altitude of $840 \mathrm{~m}$ with the CNES provided rocket motor. The body and fins are made of CFRP (Carbon Fiber Reinforced Plastic), while the nose is made from GFRP (Glass Fiber Reinforced Plastic). Fig. 1 shows an exterior view of the rocket.

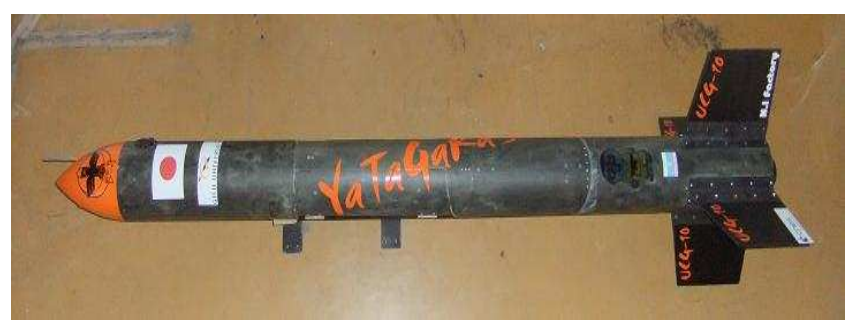

Fig. 1. Overview of UCG-10
Table 1 Major dimensions of UCG-10 Rocket

\begin{tabular}{|c|c|}
\hline Total Weight & $11.4 \mathrm{~kg}$ \\
\hline Body Lengh & $2000 \mathrm{~mm}$ \\
\hline Diameter & $150 \mathrm{~mm}$ \\
\hline Wing Span & $530 \mathrm{~mm}$ \\
\hline
\end{tabular}

On-board equipment. Fig. 2 is a cutaway view of the UCG-10 rocket showing the arrangement of onboard equipment. Nearest the nose is the avionics bay holding six circuit boards which in turn contain the pressure sensor, accelerometer and five microcomputers (dsPICs). Two video cameras and a GPS receiver are also located in this bay. Next in line is the satellite bay. It holds the quasi-satellite and a new ejection mechanism. Behind the satellite bay is the parachute bay. This bay holds a parachute, a drogue chute and a servo motor to deploy them. These are used to land expended rocket safely. The quasi-satellite carries its own parachute. Finally, at tail is the rocket motor.

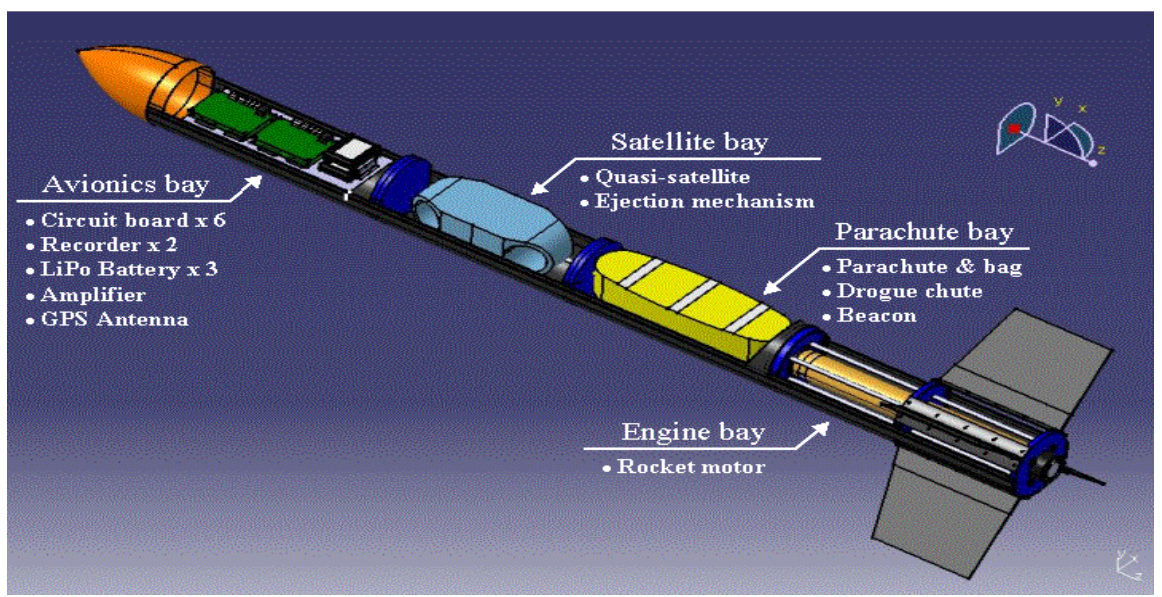

Fig. 2. Arrangement of onboard equipments

Electronics. The avionics bay contains six circuit boards containing equipment and their control facilities. A list of each board and its functions follows.

Power board: supplies power to the other boards.

Main board: controls the equipment on the other boards.

Acceleration boards: handles accelerometer data.

Pressure board: handles data from the pressure sensor.

GPS board: handles GPS data.

Communications board: sends sensor data to the ground.

A block diagram of the electronics system of the UCG-10 appears in Fig. 3. All sensor data and video footage is logged to onboard SD cards. 


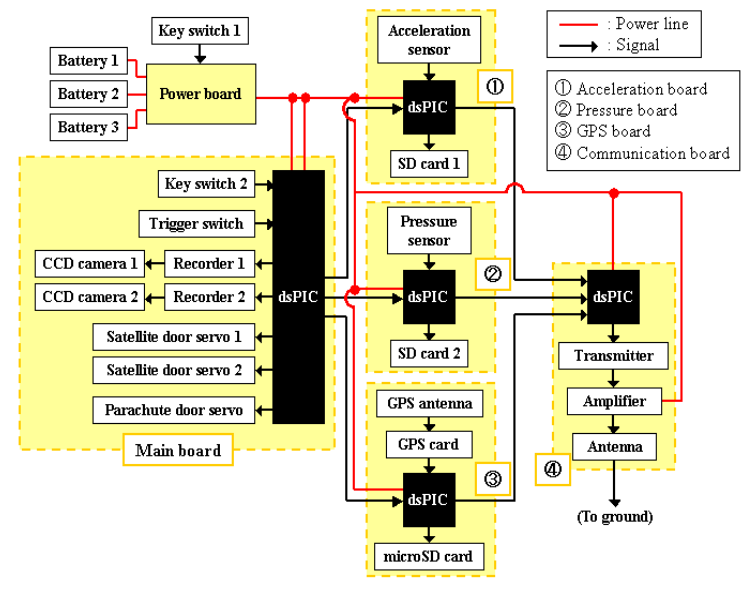

Fig. 3. Block diagram of UCG-10

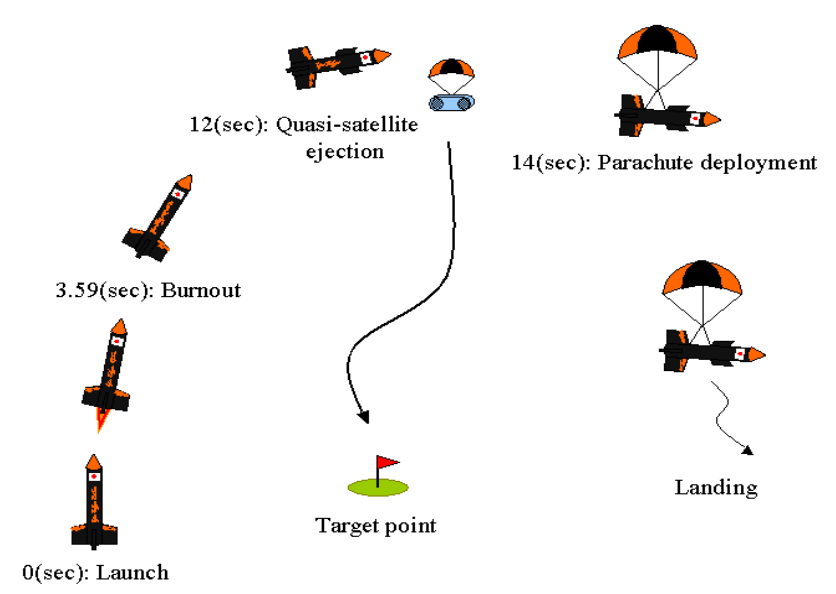

Fig. 4. Flight sequence of UCG-10

Flight sequence. The flight sequence's major events are controlled by the onboard dsPICs. A diagram of the flight sequence is shown in Fig. 4; a flowchart of events appears in Fig. 5. The rocket deploys the quasi-satellite 12 seconds after launch before engaging its own parachute 2 seconds later.
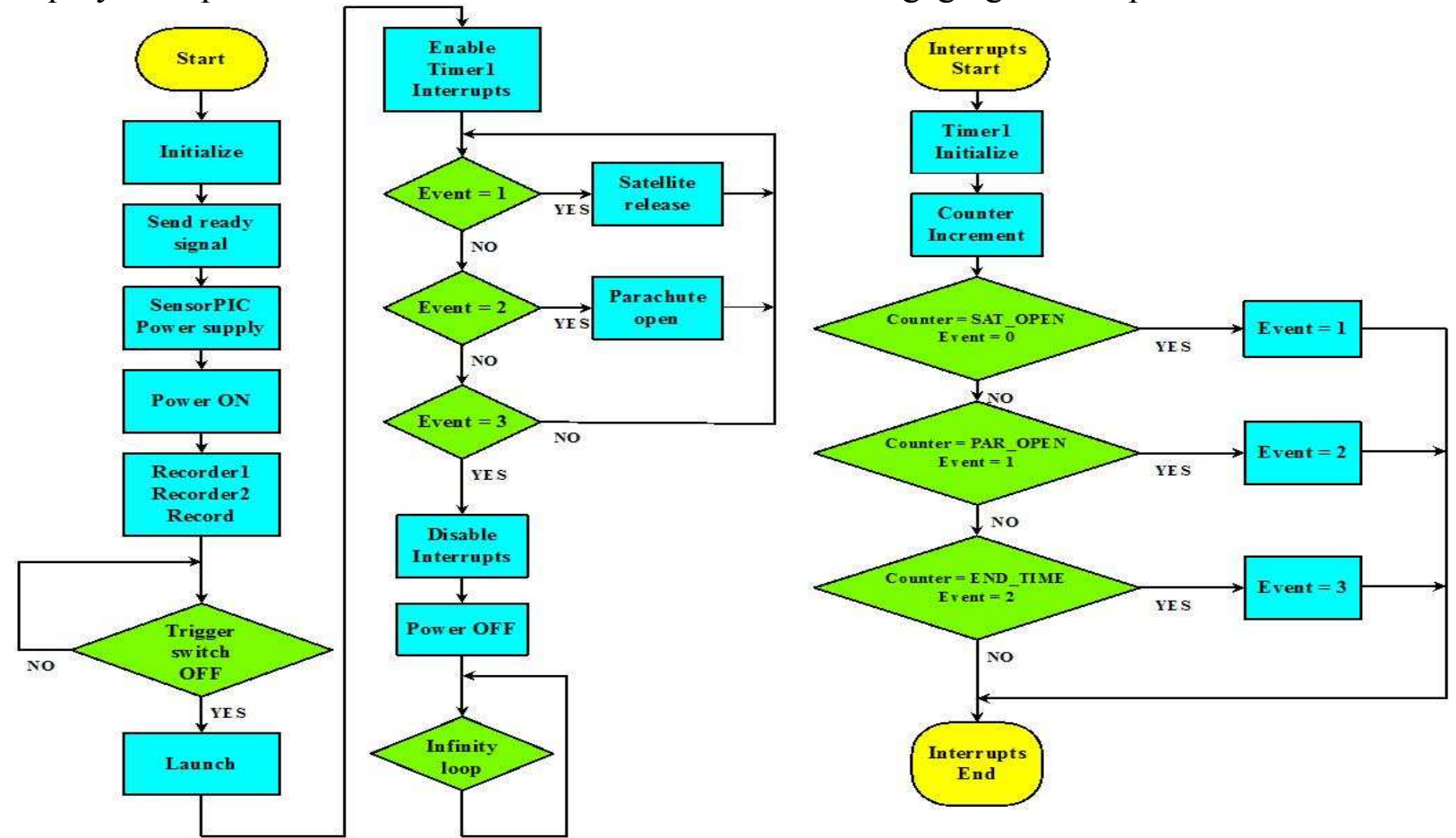

Fig. 5. Flochart of main dsPIC

\section{Quasi-Satellite}

Configuration of the quasi-satellite. The quasi-satellite is the main reason for this entire project. The rocket deploys the quasi-satellite at the peak of its trajectory. The time at which this occurs was found by calculation. After deployment, the quasi-satellite maneuvers itself to a target point using its onboard GPS receiver.

Autonomous mobile system. A limit switch lets the quasi-satellite know it has been deployed and its autonomous maneuvering program which resides in an onboard dsPIC. Using the onboard GPS receiver, the program finds its own position as well as the location of the target point. With these data and also with a record of its own previous positions, the program computes commands for the two propellers. The basic strategy is shown in Fig. 7, and a detailed flowchart is in Fig. 8. 


\section{Results of launching experiments}

Rocket results. The rocket launch went well and compared favorably with simulations we had done. The two video cameras monitored the rocket motor, the deployment of the quasi-satellite, and finally the deployment of the rocket's own parachute. The video data, as well as logs of pressure, acceleration and GPS were successfully saved to onboard SD cards.

Graphs of the flight data appeared in Fig. 9 - 14. Fig. 9 and Fig. 10 show the GPS data and the acceleration data respectively for the UCG-10 rocket. Fig. 11 shows the experimental and simulated $\mathrm{Z}$-axis acceleration data together for comparison. The graphs coincide closely and gives us confidence in the sensor data. An image of the quasi-satellite deployment appears in Fig. 14.

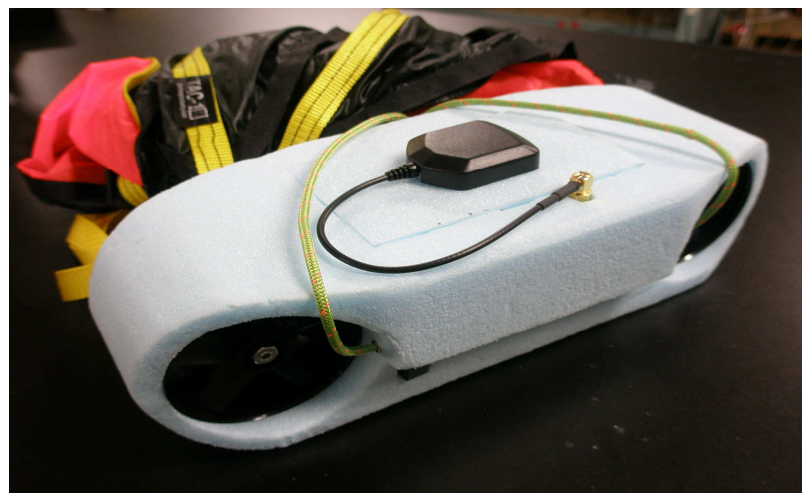

Fig. 6. Overview of the quasi-satellite

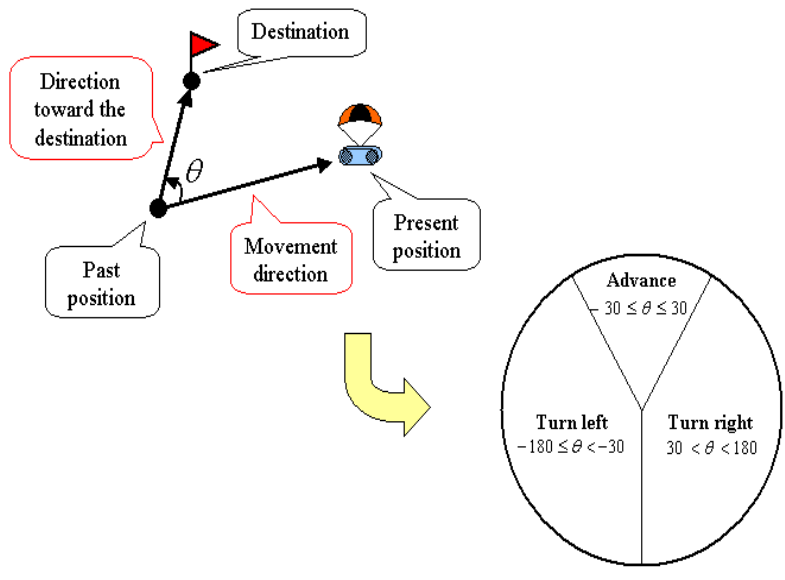

Fig. 7. Autonomous moving system

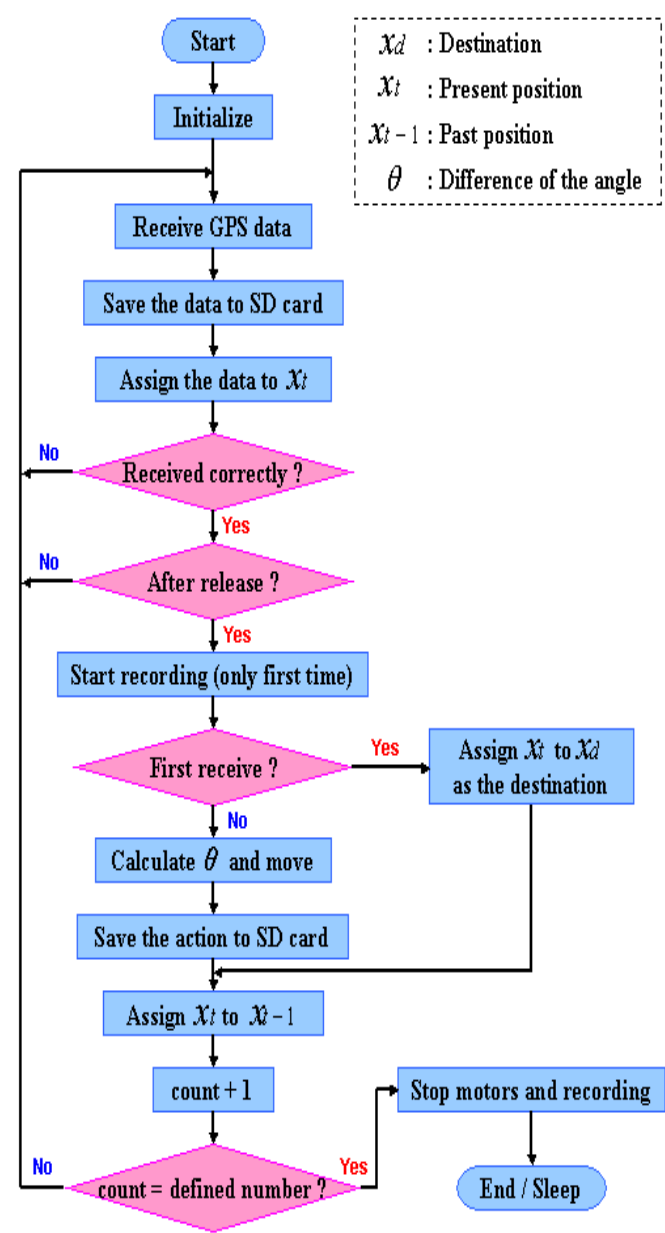

Fig. 8. Flowchart of the quasi-satellite

Quasi-Satellite results. The quasi-satellite deployed successfully and was recovered intact. Unfortunately, due to high winds on launch day, it was impossible to maneuver to the target point. Fig. 12 and Fig. 13 shows the GPS data recorded onboard the quasi-satellite.

\section{Additional experiments}

We performed additional experiments to confirm the operation of the quasi-satellite's autonomous maneuvering system. In these experiments, the quasi-satellite rode on a three-wheeled cart. The results appear in Fig. 15 and Fig. 16, where a yellow rectangle indicates the starting position and the red dot shows the target point. Fig. 15 shows the results of starting with cart facing away from the target. Fig. 16 shows the results of starting with the target on one side of the cart. 


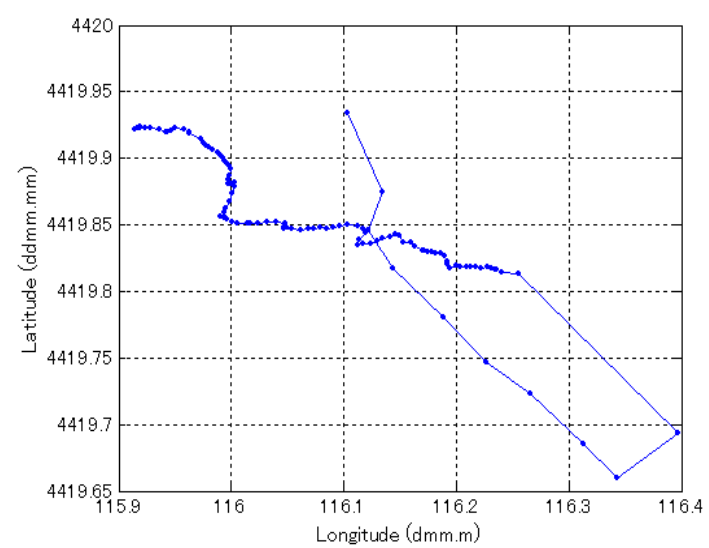

Fig. 9. Transmitted GPS data of the rocket

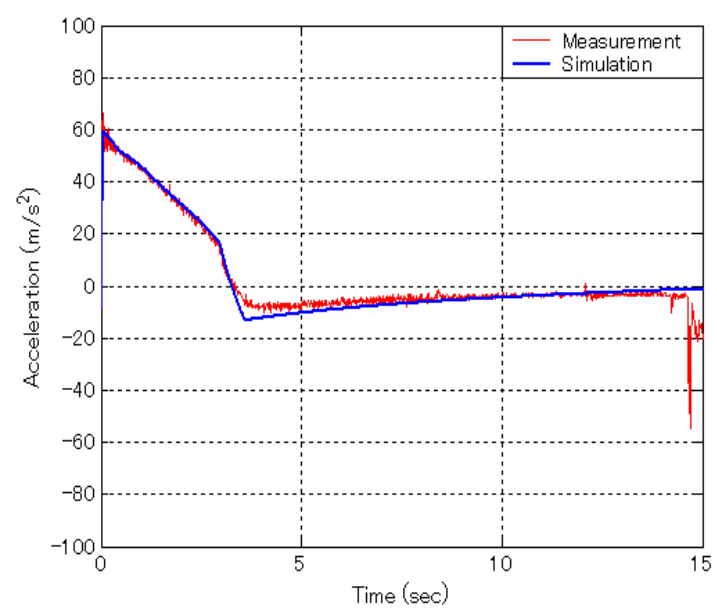

Fig. 11. The comparison of acceleration

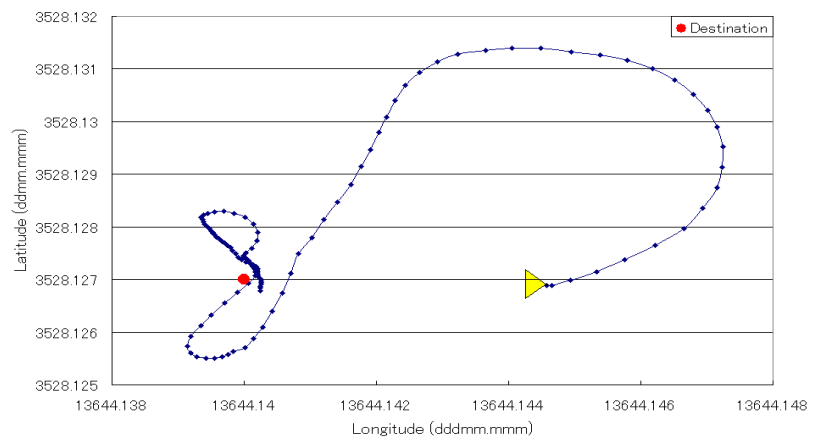

Fig.15. Satellite path from take off to Destination (Start from a forward point)

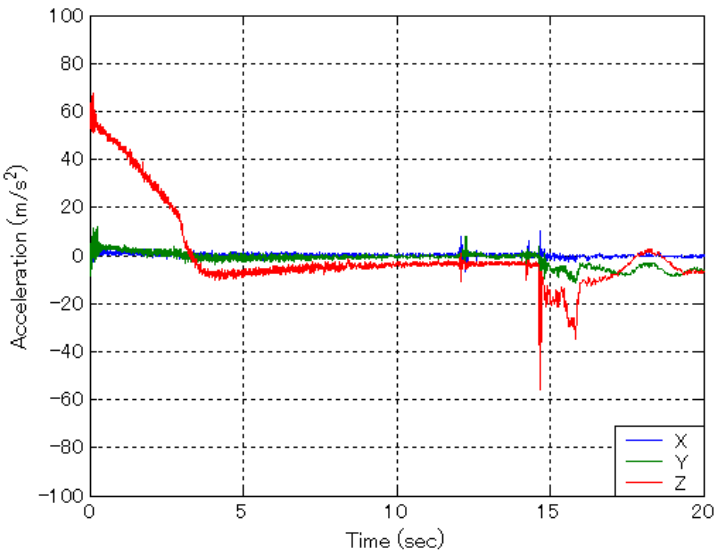

Fig. 10. Experimental result of acceleration data

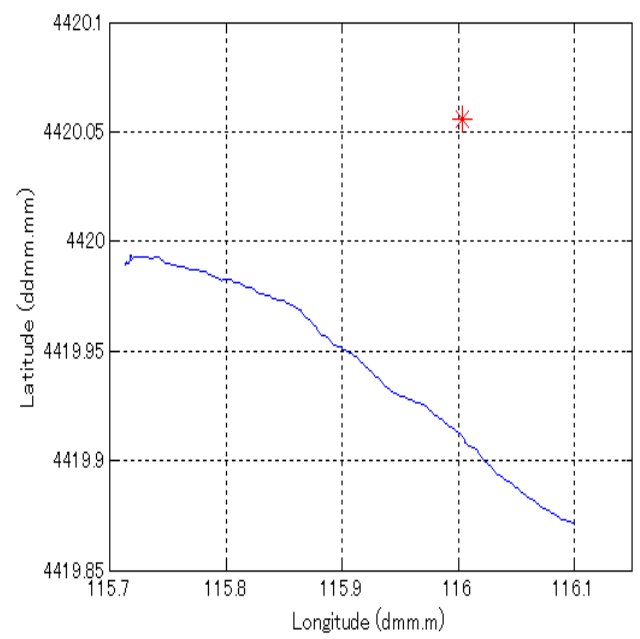

Fig. 12. Latitude and longitude of the satellite GPS

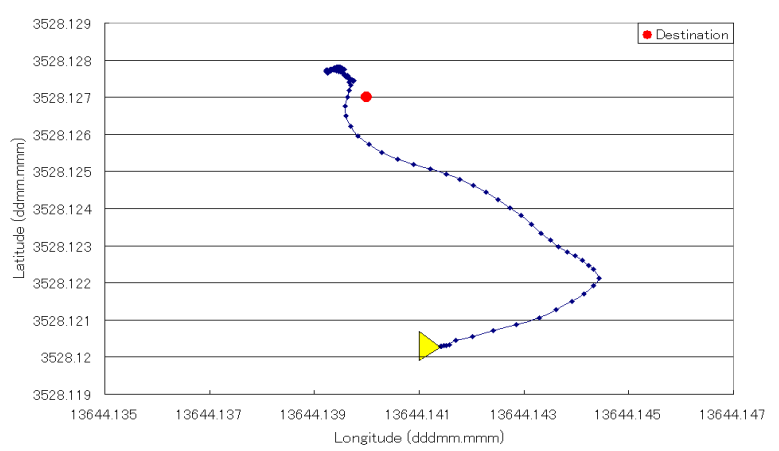

Fig.16. Satellite path from take off to Destination (Start from a right point)

\section{Conclusion}

This paper presented the system development of a small experimental rocket and a quasi-satellite for the French launch campaign conducted by the Association of Planete Sciences in 2010. The campaign provides a setting for learning and experiencing the conducting of experiments and manufacturing under rigorous project management.

A rocket named the UCG-10 was developed which included onboard sensors for pressure and acceleration and a GPS receiver. The sensor data was stored onboard for later comparison with simulation data. The rocket deployed a quasi-satellite at the peak of its trajectory. The quasi-satellite has its own GPS receiver and maneuvering propellers and is to direct itself to land at a target point. 
High winds on launch day prevented this from happening, but all data was successfully logged and its close correspondence with simulation results gives us confidence in the strategy. This paper has successfully demonstrated the use of adequate control mechanism in the controlling of a satellite.

\section{Acknowledgements}

This work has been created by the members of 'Space Club-Gifu' and Sasaki Lab. in Gifu University. The club has always been invited by the French Association of Planete Sciences to participate in their launching campaign at the DGAEM, Biscarrosse in France since 2008. Furthermore this program has been financially supported by a private company "Kakamigahara koukuuki". We would like to express our sincere thanks for their help and support.

\section{References}

[1] http://www.planete-science.org/.

[2] Book of Specification Single Stage Experiment Rockets (Version 2.1), Planete Sciences/CNES (Centre National d'Etudes Spatiales), issued on $15^{\text {th }}$ October, 2004.

[3] KIWI-Millenium Telemetry System Comprehensive Data Sheet, Planete Sciences/CNES (Centre National d'Etudes Spatiales), issued in May, 2007.

[4] Minoru Sasaki, Noriaki Nakano and Satoru Ohmayu, "System development of an experimental rocket for launching campaign organized by French association Planete Sciences",ISTS2008-u-07, 2008

[5] Christophe SCICLUNA, Kenji OGIMOTO, Minoru SASAKI and Koichi YONEMOTO, "THE FRENCH NATIONAL ROKETS LAUNCHING CAMPAIGN AND THE DAWN OF ITS COLLABORATION WITH JAPANESE AMATEUR SPACE CLUBS”, ISTS 2008-u-06, 2008

[6] Naoki OGUSHI, Minoru SASAKI, Satoshi ITO, Noriaki NAKANO, Akihiro ASAI, Naoya OZEKI, Norio IKEDO, Hansang LEE, "Development of a computer controlled experimental rocket and an autonomous mobile quasi-satellite using PICs", Journal of the Japan Society of Applied Electromagnetics and Mechanics, 17(2), 2009, pp. 360-366. 